\title{
Calling amplified haplotypes in next generation tumor sequence data
}

\author{
Ninad Dewal, ${ }^{1}$ Yang Hu, ${ }^{2}$ Matthew L. Freedman, ${ }^{3,4}$ Thomas LaFramboise, ${ }^{5}$ \\ and Itsik $\mathrm{Pe}^{\prime} \mathrm{er}^{2,6}$ \\ ${ }^{1}$ Department of Biomedical Informatics, Columbia University, New York, New York 10032, USA; ${ }^{2}$ Department of Computer Science, \\ Columbia University, New York, New York 10027, USA; ${ }^{3}$ Department of Medical Oncology, Dana-Farber Cancer Institute, Boston, \\ Massachusetts 02115, USA; ${ }^{4}$ Medical and Population Genetics Program, The Broad Institute of Harvard and MIT, Cambridge, \\ Massachusetts 02142, USA; ${ }^{5}$ Department of Genetics, Case Western Reserve University School of Medicine, Cleveland, Ohio 44106, \\ USA
}

\begin{abstract}
During tumor initiation and progression, cancer cells acquire a selective advantage, allowing them to outcompete their normal counterparts. Identification of the genetic changes that underlie these tumor acquired traits can provide deeper insights into the biology of tumorigenesis. Regions of copy number alterations and germline DNA variants are some of the elements subject to selection during tumor evolution. Integrated examination of inherited variation and somatic alterations holds the potential to reveal specific nucleotide alleles that a tumor "prefers" to have amplified. Next-generation sequencing of tumor and matched normal tissues provides a high-resolution platform to identify and analyze such somatic amplicons. Within an amplicon, examination of informative (e.g., heterozygous) sites deviating from a 1:1 ratio may suggest selection of that allele. A naive approach examines the reads for each heterozygous site in isolation; however, this ignores available valuable linkage information across sites. We, therefore, present a novel hidden Markov model-based method-Haplotype Amplification in Tumor Sequences (HATS) - that analyzes tumor and normal sequence data, along with training data for phasing purposes, to infer amplified alleles and haplotypes in regions of copy number gain. Our method is designed to handle rare variants and biases in read data. We assess the performance of HATS using simulated amplified regions generated from varying copy number and coverage levels, followed by amplicons in real data. We demonstrate that HATS infers the amplified alleles more accurately than does the naive approach, especially at low to intermediate coverage levels and in cases (including high coverage) possessing stromal contamination or allelic bias.
\end{abstract}

[Supplemental material is available for this article.]

Tumor development and growth can be viewed as an evolutionary process (Nowell 1976). Genetic variation in the form of somatic alterations (e.g., mutations, translocations) and inherited polymorphisms provide the raw material for the acquisition of tumorrelated traits. Copy number aberrations (CNAs)—regions of somatic amplification (amplicons) or deletion-are a hallmark of tumor genomics. Recurrent amplicons have been observed over two decades (Kallioniemi et al. 1992; Joos et al. 1995; Cher et al. 1996; Korn et al. 1999; Paris et al. 2004; Zhao et al. 2005; Sun et al. 2007a) and are believed to be advantageous to the tumor during tumor development.

Genome-wide scans of CNAs have progressed in resolution from technologies such as traditional comparative genomic hybridization (CGH) to array-based CGH (Solinas-Toldo et al. 1997; Bentz et al. 1998), including tiling array CGH (Ishkanian et al. 2004) and single nucleotide polymorphism (SNP) arrays (Wang et al. 1998; Lin et al. 2004); methods for copy number detection on such platforms oftentimes utilize hidden Markov models (HMMs; see Supplemental Methods). The recent advent of high-throughput, next generation sequencing (NGS) platforms now offers tremendous opportunities in characterizing genomes-healthy or disease-affected-at the nucleotide level of resolution.

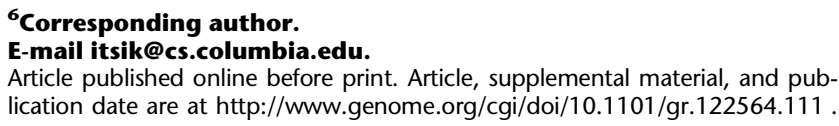

Modern sequencing of genomes is massively parallel. The subject's DNA is first sheared, after which the laboratory-amplified fragments are sequenced, producing short reads that are several dozen bases long (Ronaghi et al. 1996; Gharizadeh et al. 2002; Bentley et al. 2008; McKernan et al. 2009). Reads are aligned to the human reference genome (Bentley et al. 2008; Li et al. 2008a, b; Alkan et al. 2009; Langmead et al. 2009; Li and Durbin 2009; McKernan et al. 2009). Since reads at a site are assumed to sample each of its two original copies (or more, in the case of amplicons), multiple independent reads can be observed to cover each haploid copy of a site in a manner following the Poisson distribution with genome-wide expectation $\Lambda$ (as determined by the laboratory amplification step).

Multiple computational approaches for detecting structural variants and copy number changes within NGS data have been developed (Dalca and Brudno 2010). One such class of methods utilizes paired-end sequence information for detection of germline insertions, deletions, and inversions (Tuzun et al. 2005; Korbel et al. 2007; Bentley et al. 2008; Chen et al. 2009; Hormozdiari et al. 2009; Lee et al. 2009; McKernan et al. 2009). Another such class examines depth of reads to infer germline copy number variants (CNVs) (Xie and Tammi 2009; Yoon et al. 2009). This read depth paradigm was also applied to tumor and matched normal tissues to detect copy number and breakpoints of CNAs in tumors (Chiang et al. 2009). A third class combines read depth with mate pairs for CNV calling (Medvedev et al. 2010).

In addition to CNAs, inherited polymorphisms are clearly related to cancer biology and predisposition. Classic examples in- 
clude the highly penetrant mutations in BRCA1 (Hall et al. 1990; Miki et al. 1994) and BRCA2 (Wooster et al. 1995) that lead to breast cancer. More recently, genome-wide association studies (GWAS) have led to the discovery of more modestly penetrant variants that are associated with human traits (McCarthy et al. 2008; Hindorff et al. 2009; Witte 2010), including cancer susceptibility (Amundadottir et al. 2006; Freedman et al. 2006; Zanke et al. 2007; Amos et al. 2008; Easton and Eeles 2008; Fletcher et al. 2008; Hung et al. 2008; Thorgeirsson et al. 2008; Ahmed et al. 2009; Le Marchand 2009; Song et al. 2009; Wu et al. 2009; Chung et al. 2010; Stadler et al. 2010a, b; Turnbull et al. 2010).

GWASs stem partially from modern population genetics, which offers ample data and models to understand sequence polymorphisms - mostly single nucleotide variants (SNVs) — along with their correlation to one another and to disease phenotypes (Hartl and Clark 2007). Specifically, the nonrandom allele combinations of proximal SNVs along a single genomic copy, called haplotypes, are a useful unit of local genomic variation. Although haplotypes are not observed directly from genotype data, computational phasing methods (Kimmel and Shamir 2005; Rastas et al. 2005; Eronen et al. 2006; Scheet and Stephens 2006; Browning and Browning 2007; Sun et al. 2007b) distinguish maternal from paternal alleles, thus reconstructing germline haplotypes. Amplicons in cancer typically lie along a haplotype.

Since the somatic genome is a descendent of the germline genome, recent studies have explored the relationships between these distinct but related genomes (Jones et al. 2009; Kilpivaara et al. 2009; Olcaydu et al. 2009). For example, a particular heterozygous locus in a tumor may "prefer" to have one germline allele somatically amplified over another. Such an event has been demonstrated in a targeted fashion in mouse skin tumors (Nagase et al. 2003; de Koning et al. 2007) and in human colorectal cancers (Ewart-Toland et al. 2003; Hienonen et al. 2006). The latter studies found the AURKA gene to be preferentially amplified when containing a low penetrance $(\mathrm{T}>\mathrm{A})$ germline variant. In order to robustly perform this type of analysis genome-wide, allelic copy number status must first be measured; several existing algorithms do this on SNP arrays (Nannya et al. 2005; Komura et al. 2006; Laframboise et al. 2007; Korn et al. 2008). We recently reported such an analysis and discovered new links between germline SNP variants within somatic amplicons in glioblastoma SNP array data (Dewal et al. 2010; LaFramboise et al. 2010). The higher resolution, coverage, and larger dynamic range of NGS platforms now compel us to address such questions on tumor sequence data. As a first step, we must determine allelic copy number status of the reference alleles and SNVs within amplicons.

We present a novel method for analyzing NGS data in order to distinguish the amplified from the nonamplified alleles within tumor CNA regions, which themselves can be identified beforehand from the same data. We assume that only one of the chromosomes in a homologous pair undergoes amplification along an amplicon, as the majority of amplifications were observed to be monoallelic versus biallelic in earlier work (LaFramboise et al. 2005). As we later show, the statistical signal for allelic imbalance of amplification that is coming from a single heterozygous site is often inconclusive due to limitations of coverage, sequencing bias, and stromal contamination. We, therefore, collate information from multiple heterozygous sites by leveraging the known structure of linkage disequilibrium (LD) between these variants within the population being interrogated. Specifically, we develop an HMM-based approach, called Haplotype Amplification in Tumor Sequences (HATS), that reports the amplified alleles, and thus haplotypes, in the tumor sample based on (1) coordinates and copy number of CNA regions in a tumor sample called by existing methods, (2) allele-specific counts of reads from tumor and matched normal sequences (when available) corresponding to those regions (Li et al. 2009), (3) genotype calls of sites within those regions, and (4) independent training data consisting of phased haplotype sequences from the same population as that of the sample. This training data provides LD information across sites, allowing for more accurate haplotype construction versus examining each site in isolation. In contrast to prior work based on SNP array data (Dewal et al. 2010), HATS is able to handle information unique to sequencing, such as rare or low frequency variants, including novel SNV sites or somatic mutations not represented in the training data. Evaluation of HATS using synthetic data sets as well as real tumor data, obtained from The Cancer Genome Atlas (TCGA) (Network 2008), emphasizes that HATS detects the amplified allele (within called amplicons) more accurately than an alternative, naive approach over $99 \%$ of the time. The gain is especially prominent at lower to intermediate levels of average coverage, as well as in cases (including high coverage) involving stromal contamination or allelic bias.

\section{Results (Evaluation)}

For each heterozygous site within a called amplicon $a$ (of copy number $C_{a}$ ) in a tumor sample, the naive model compares the counts of reads that observe each allele and designates the allele with the greater read count as the amplified allele (see Methods). If the read counts are equal, no call is made. The naive model is thus vulnerable to allele-specific biases in addition to fluctuations in read counts that occur at low coverage levels. HATS is designed to address these issues. HATS examines the allele-specific read depth and calculates allelic biases for each site within $a$, along with leveraging known LD structure over multiple sites (see Methods), to call the amplified alleles.

The advantage that this provides to HATS must be gauged. We summarize the performance of both the naive model and HATS using the metric sensitivity, or the probability of a gold standard amplified allele at a heterozygous site being correctly called as amplified. We examine only those sites within regions known to be amplified, called a priori by a copy number-calling algorithm or a different platform such as array CGH. Thus, specificity, in this case-the fraction of nonamplified alleles (within an amplicon) called as nonamplified-is identical to sensitivity. We first derive the power of the naive model theoretically. Afterward, we determine the sensitivity of the naive model and HATS using simulated data, followed by real data.

\section{Theoretical power of the naive model}

The number of reads that cover each haploid copy of a site follows the Poisson distribution with genome-wide expectation $\Lambda$. At a heterozygous site, $\Lambda$ and $\left(C_{a}-1\right) \times \Lambda$ represent the mean read counts for the nonamplified and amplified alleles, respectively. The combined mean for both alleles is $\Lambda+\left(C_{a}-1\right) \times \Lambda=C_{a} \times \Lambda$, and the diploid coverage is $2 \Lambda$. The theoretical power of the naive model is the total probability of the amplified allele possessing a read count greater than that of the nonamplified allele, with the space of read count pairs generated from Poisson curves with respective means just mentioned. This is described more formally in Supplemental Methods. Results over a range of values for $2 \Lambda$ and $C_{a}$ are shown in Supplemental Figure S1. 


\section{Performance of HATS and the naive model in simulations}

To measure the sensitivity of HATS across a variety of tumor data set scenarios, we generated numerous synthetic data sets containing amplicons and assessed HATS' ability to call the amplified alleles within the amplicons. We performed the same for the naive model as a baseline comparison. Simulations revealed that HATS' sensitivity eclipses that of the naive model over $99 \%$ of the time in practical data sets.

In further detail, simulation of a particular data set first requires training data consisting of phased germline genotypes for $d$ unrelated individuals from the same population. We select $n$ of the $d$ individuals to comprise the test data (indexed by $1 \leq j \leq n$ ). Stretches of somatic amplification are randomly generated and applied along the genome of each sample $j$ such that these recurrent stretches overlap across the samples. Each CNA amplicon $a$ in $j$ thus consists of a gold-standard amplified and nonamplified haplotype pair. For a heterozygous genotype at a site in $a$, counts of reads that observe the amplified allele and nonamplified allele are sampled from Poisson distributions with respective mean haploid coverages $\left(C_{a}-1\right) \times \Lambda_{j}$ and $\Lambda_{j}$. For a homozygous site in $a$, counts of reads that observe the allele are sampled from a Poisson distribution with mean haploid coverage $C_{a} \times \Lambda_{j}$. In the scenarios that incorporate allelic bias (described later), these read counts may be adjusted to reflect the simulated bias.

For each sample $j$, the allele-specific read count information along each amplicon in $j$ is analyzed by the naive model. In addition, the training data of $(d-n)$ samples, along with genotype information, copy number $C_{a}$, tumor allelic read counts, and normal allelic read counts (for calculating bias) along each amplicon in $j$ are analyzed by HATS. We define accuracy as the fraction of gold-standard amplified alleles along heterozygous sites in each simulated CNA region $a$ that is correctly called as amplified.

Setting $n>1$ is only relevant for scenarios in which allelic bias is simulated, as multiple samples provide a better estimate of the bias. When not simulating bias, we set $n=1$ and employ a $d$-fold cross-validation scheme in which each $a$ in each $j$ is processed by the naive method and HATS, the latter using a training data set of $(d-1)$ samples. Again, overlapping amplicon coordinates are applied to each $j$.

The variability across the synthetic data sets is implemented via a set of seven parameters, described in Table 1. We perform 100 trials for each parameter value combination when iterating over the parameter space, performing a $d$-fold cross-validation per trial if $n=1$. To prevent an explosive growth of the parameter space, we iterate over only one parameter at a time, while maintaining the other parameters at their default values. The exception to this is when we simulate bias, in which we explore the space of the last two parameters, as described later.

Toward determining sensitivity for a parameter value combination, we focus only on those accuracies (from the combination's trials) whose corresponding amplicons cover at least a threshold of $v$ heterozygous sites. An example plot of accuracies for each amplicon $a$ versus the number of heterozygous sites in $a$ is depicted in Figure 1A (using example parameter values $\left[2 \Lambda_{j}=6, C_{a}=3\right]$ ). Note that as the number of heterozygous sites in $a$ increases, the accuracies converge to a peak for either method. The peak thus represents an asymptotic measure for accuracy, which we assign as the sensitivity. A large value for $v$ isolates those points contributing to the peak while avoiding the discreteness effects observed in small values. To determine the value of each peak, we perform $k$-means clustering on those points passing $v$,

Table 1. Simulation parameter definitions

\begin{tabular}{|c|c|c|}
\hline Parameter name & Default & Description \\
\hline Amplicon copy number $\left(C_{a}\right)$ & 3 & $\begin{array}{l}\text { This parameter represents the number of haploid copies of the genome along amplicon } \\
a \text { in tumor sample } j \text {. }\end{array}$ \\
\hline $\begin{array}{l}\text { Haploid genome-wide coverage of } \\
\text { tumor }(\Lambda)\end{array}$ & 5 & $\begin{array}{l}\text { This parameter represents the mean of a Poisson distribution which, upon sampling, } \\
\text { determines the number of reads on a haplotype at a particular site } i \text { in tumor sample } j \text {. }\end{array}$ \\
\hline $\begin{array}{l}\text { Haploid genome-wide coverage of } \\
\quad \text { normal }(\tilde{\Lambda})\end{array}$ & 5 & $\begin{array}{l}\text { This parameter represents the mean of a Poisson distribution which, upon sampling, } \\
\text { determines the number of reads on a haplotype at a particular site } i \text { in the matched } \\
\text { normal of sample } j \text {. }\end{array}$ \\
\hline Mean length of a recurrent amplicon & $390 \mathrm{~kb}$ & $\begin{array}{l}\text { This parameter represents the mean of an exponential distribution which, upon sampling, } \\
\text { determines the length of recurrent amplicons across samples. The distribution possesses } \\
\text { a mean of } 390 \mathrm{~kb} \text { by default. This exponential distribution can produce stretches of } \\
\text { over } 1 \text {, or even } 2, \mathrm{Mb} \text {. The default value was determined in Dewal et al. (2010). }\end{array}$ \\
\hline Number of recurrent amplicons & 5 & $\begin{array}{l}\text { This parameter determines the number of recurrent amplicons in the genome. A value } \\
\text { of } 5 \text { represents a realistic number of such regions, as was determined in Dewal et al. } \\
(2010) \text {. }\end{array}$ \\
\hline GC read bias ratio & 1.0 & $\begin{array}{l}\text { This parameter is used to represent GC bias that is observed in real sequence data. It } \\
\text { represents the ratio of the simulated AT read count to the simulated GC read count at } \\
\text { the site } i \text { in question. The idea is that the presence of a } G \text { or } C \text { at } i \text { translates to a } \\
\text { (slightly) higher GC content level, which may disrupt the sequencing chemistry and } \\
\text { thus induce bias. A value of } 1.0 \text { indicates no bias, while larger values indicate stronger } \\
\text { bias. The default value is set to } 1.0 \text { so that other parameters can be tested } \\
\text { independently of bias during simulation. }\end{array}$ \\
\hline Number of samples in test data & 1 & $\begin{array}{l}\text { This parameter represents the number of samples that are to be excised from the training } \\
\text { data set in order to be used as test data. For example, if the original training data set } \\
\text { contained ten individuals, and this parameter was set to } 2, \text { then two individuals would } \\
\text { comprise the test data, while eight would comprise the effective training data to be } \\
\text { used in the simulations. The default value is set to } 1 \text {, as values }>1 \text { are only relevant when } \\
\text { simulating with bias. Increasing the test data size in bias simulation improves HATS' } \\
\text { estimation of the bias. }\end{array}$ \\
\hline
\end{tabular}

Two of the default values were obtained by observing parameter-specific properties in a real Illumina 550K data set obtained from The Cancer Genome Atlas (TCGA), published from a previous study (Dewal et al. 2010).

\section{Genome Research}

www.genome.org 


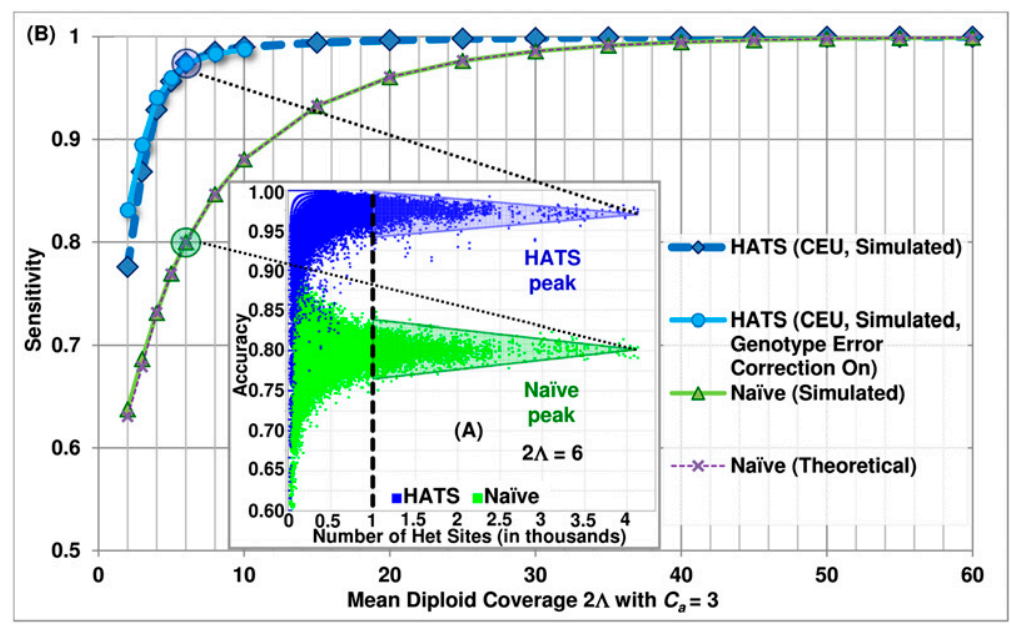

Figure 1. Accuracy example and sensitivity of HATS and the naive method from simulations, European (CEU) training sata set. (A) Accuracies of each sample trial $\left(2 \Lambda_{j}=6, C_{a}=3\right)$. Each point in the embedded, raised-dot plot represents the accuracy for a particular amplicon $a$ in sample $j$ per trial. As the number of heterozygous sites in $a$ increases, the accuracies converge to a peak for the naive method and a peak for HATS. We set the threshold $v$ to 1000 and use $k$-means clustering to determine the centroids for each peak. The centroid for the naive model resides at $\sim 0.80$, which is assigned as the sensitivity for the naive method for parameter values $\left(2 \Lambda_{j}=6, C_{a}=3\right)$. The centroid for HATS exists at $\sim 0.975$. (B) Method sensitivities. This figure displays the simulation sensitivity results for HATS (with Genotype Error Correction [GEC] turned on or, by default, off) as well as for the naive method. The naive theoretical curve is included for comparison purposes, illustrating that the naive results can, indeed, be calculated theoretically. Note that it takes up to diploid coverage of 45 until the naive method can match the performance of HATS. The GEC mode noticeably improves performance at very low coverage levels for HATS. The training data set was obtained from the 1000 Genomes Project (http://www.1000genomes.org/). performs it in $93.4 \%$ of points. Example plots depicting accuracy over varying numbers of heterozygous sites across varying diploid coverage levels are given in Supplemental Figure S2. Points encompassing at least $v=1,000$ heterozygous sites converge to their associated peak, which indicates sensitivity.

The sensitivities of the two methods across varying levels of coverage (with a default copy number of 3 ) in the European individuals are depicted in Figure 1B. Both curves for HATS - with a Genotype Error Correction mechanism either enabled for low $2 \Lambda_{j}$ (to recover an allele possibly missed due to no reads observing that allele at low coverage; see Supplemental Methods) or disabled-perform better than the naive model, especially at intermediate to lower coverage levels. We also show that the naive simulated sensitivity is congruent with its theoretical estimate. For both methods, the breakdown of sensitivities per read count observing a site over the coverage levels is given in Supplemental Figure S3. Sensitivities for Yoruban and Asian individuals are depicted in Supplemental Figure S4 and show similar performance. setting $k=1$ for each method. The resulting centroid for each method represents the peak and thus the sensitivity for that method.

\section{Simulation results}

We obtained three training data sets from the 1000 Genomes Project (The 1000 Genomes Project Consortium 2010), each of which contained phased haplotype sequences from individuals from a HapMap population (The International HapMap Consortium 2005). Any trio children were removed to preserve independence among individuals, resulting in the data sets respectively including $d=55$ European (two trio children removed), $d=55$ Yoruban (one trio child removed), and $d=59$ Japanese and Chinese individuals. These data sets were used independently to avoid stratification effects. Data for additional individuals are expected to be available publicly over time. The HATS method, as well as the evaluation procedure above, can easily work with an expanded training set.

When simulating without bias, we observed that the first two parameters (regarding amplicon copy number and tumor haploid coverage) have the most impact on sensitivity. The fourth parameter, Mean Length of a Recurrent Amplicon, increases the number of heterozygous sites in $a$ by virtue of increasing the span of $a$. We noted above that a longer $a$ provides a better estimate of the asymptotic accuracy of each method. In Figure 1A, HATS performs better than the naive method in over $99.7 \%$ of points (representing amplicons) that each encompass at least $v=20$ heterozygous sites. At the default parameter values, HATS does better in $99.3 \%$ of points that each encompass at least $v=20$ heterozygous sites, with an average accuracy gap of $10.2 \%$. When there is no restriction on $v$, HATS matches the naive method in $6.3 \%$ of points and out-

\section{Simulation results: Modeling biases}

Real sequence data is known to contain bias, a common example of which is GC bias (Bentley et al. 2008; McKernan et al. 2009). While HATS' bias correction is designed to handle allele-specific biases in read counts in general, we test its ability to handle GC biases specifically. When simulating with bias, we vary the two parameters, GC Read Bias Ratio and Number of Samples in Test Data. The resulting sensitivities are depicted in Figure 2A. The former parameter models GC bias by representing the ratio of AT reads versus GC reads at a heterozygous site. The greater the ratio, the stronger the induced bias; a value of 1.0 signifies no induced bias. Note that this parameter only affects sites $\{\mathrm{G} / \mathrm{A}, \mathrm{G} / \mathrm{T}, \mathrm{C} / \mathrm{A}, \mathrm{C} / \mathrm{T}\}$. The latter parameter determines the test data size $n$, which, when increased, improves HATS' estimate of the bias and thus the performance.

The figure depicts the performance of the naive model, HATS without bias correction ( $n=1$ is sufficient as $n>1$ is relevant only when estimating bias), and HATS with bias correction. Our method consistently outperforms the naive model. Furthermore, bias correction becomes more effective as either the level of induced bias or $n$ increases. When the simulated bias is weak and $n>1$, bias correction performs only slightly less than does no bias correction by a 0.003 cost in sensitivity. However, bias correction quickly gains the upper hand as the simulated bias increases past 1.5 (or 2 in the case of $n=2$ ). When $n=1$, the estimate of bias is not as precise and results in a slightly weaker result (with a 0.008 sensitivity cost on average) unless the induced bias is very strong (3.33). Note that there is only a marginal performance improvement when increasing $n$ from 15 to 20 . The reasons are that the improvement in estimating the bias plateaus and that the training data set size is reduced, which negatively impacts sensitivity. The latter reason may be assuaged with a larger $d$. In general, the figure demonstrates 

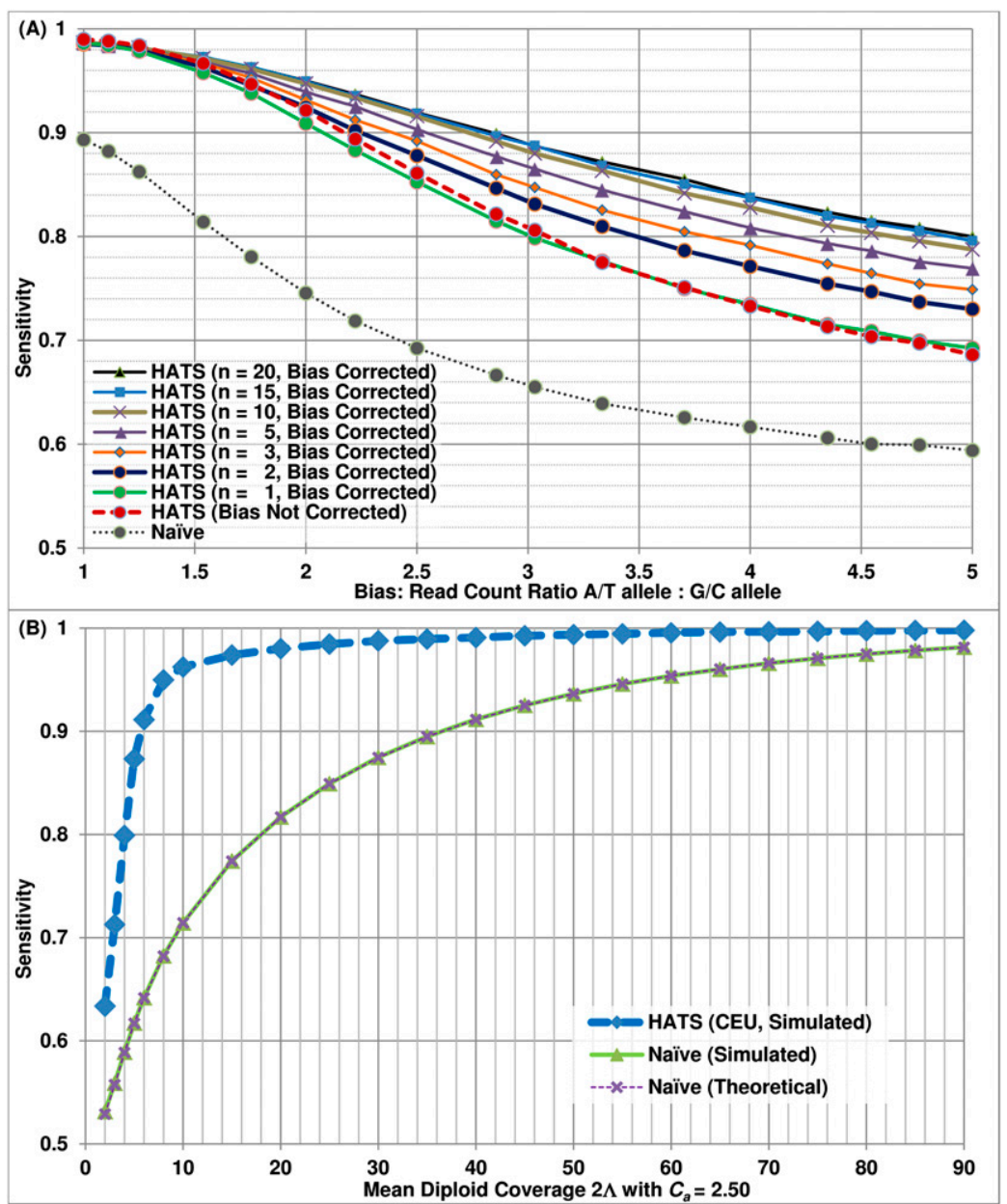

Figure 2. Results of simulated aspects of real tumor data. $(A)$ Sensitivity of HATS with bias correction and the naive model from simulations, European (CEU) training data set. This figure displays the simulation sensitivity results for HATS with bias correction activated (using varying sample sizes to estimate bias), bias correction inactivated, and the naive model. The $x$-axis represents the simulated induced bias, ranging from 1.0 (representing no bias) to 5 (representing strong bias). HATS eclipses the naive method in all instances. When the simulated bias is weak and $n>1$, bias correction performs only slightly less than does no bias correction by a 0.003 cost in sensitivity. However, bias correction quickly gains the upper hand as the simulated bias increases past 1.5 (or 2 in the case of $n=2$ ). When $n=1$, the estimate of bias is not as precise and results in a slightly weaker result (with a 0.008 sensitivity cost on average) unless the induced bias is very strong (3.33). Note that there is only a marginal improvement in performance when increasing the test data set size from 15 to 20 . The reason is that this reduces the training data set size, which negatively impacts sensitivity; in addition, improvement in estimation of the bias plateaus. (B) Sensitivity of HATS and the naive model from simulations with stromal contamination, European (CEU) training data set, copy number 2.5. This figure displays the simulation sensitivity results for HATS as well as for the naive model, given a copy number of 2.5 , which represents a tumor of copy number 3 with $50 \%$ stromal contamination of copy-neutral healthy cells. Note that the performance gap between the two methods remains wide, and the naive method does not catch up to HATS even at a very high diploid coverage of 90 . The training data set was obtained from the 1000 Genomes Project (http:// www.1000genomes.org/).

that HATS can accommodate and correct for stronger biases with the tradeoff of performing slightly weaker-a loss of 0.003 in sensitivity for $n>1$-for sites possessing smaller biases.

\section{Simulation results: Modeling stromal contamination}

In tumor data, the called copy number of an amplicon often deviates from an integer quantity. The reasons are that the tumor cells may not all carry the same aberration (i.e., intra-tumoral genetic heterogeneity) and that a tumor sample may be admixed with normal cell types (i.e., stromal contamination). We focus on the latter reason. A region that is amplified in the tumor (with $C_{a}=3$ ) but copy-neutral in the healthy somatic cells may average to a noninteger copy number, e.g., 2.50 in the case of a 50-50 mixture. We extend HATS to handle noninteger copy numbers and test its performance on simulated regions with $C_{a}=2.50$ and $C_{a}=2.80$, with the respective results shown in Figure 2B and Supplemental Figure S5. Note that as coverage reduces, the sensitivity of HATS is smaller than that from equivalent coverage levels with $C_{a}=3$. However, HATS maintains its gap over the naive method. More importantly, the naive simulated and theoretical curves do not converge with HATS' curve even when coverage is high or very high (in the case of $C_{a}=2.50$ ), strongly suggesting that the naive method performs inadequately in the common scenario of imperfect tumor purity. The breakdown of (Fig. 2B) sensitivities per read count observing a site over $2 \Lambda_{j}$ is depicted in Supplemental Figure S6.

\section{Simulation results: Hemizygous deletions with stromal contamination}

We have extended HATS to analyze heterozygous deletion mixtures, in which deleted alleles are difficult to identify as lost due to the reads coming from the stromal cells that observe those alleles. HATS thus utilizes these read counts (along with LD structure) to identify these alleles (and haplotypes) as the ones lost in the tumor. HATS can analyze data with a copy number between 1.5 and 2 , exclusive. An example of simulation results (with $C_{a}=1.9$ ) is depicted in Supplemental Figure S7. A potential future extension involves handling data with pure heterozygous deletions $\left(C_{a}=1\right)$ when matched normal information is available; this functionality is already partially implemented via HATS' Genotype Error Correction feature. We do not focus on pure homozygous deletions, as the lost alleles may be recovered by examining the matched normal genotypes or via existing germline imputation algorithms (Browning and Browning 2007). From this point onward, we return to focusing on amplifications only.

\section{Performance of HATS and the naive model in real data}

We also evaluate the performance of both methods on CNA regions in real data. We consider data that has been sequenced as well as typed on an independent platform such as SNP arrays. The sequenced tumor data would be of high enough coverage $2 \bar{\Lambda}$ to

\section{Genome Research} www.genome.org 
accurately obtain genotype, copy number, and allele-specific read count information beforehand. For any amplicon $a$ with $C_{a} \geq 3$, the amplified alleles that the naive method calls within $a$ are treated as the gold standard for $a$, as simulation results in Figure 1B reveal the naive method's good performance at high coverage. Alternatively, the gold-standard amplified alleles may be called from the SNP array using, for example, B-allele frequency differences (see Methods in LaFramboise et al. [2010] for a detailed procedure and quality control filtering steps). In either case, we then down-sample a random fraction $\Lambda / \bar{\Lambda}$ of the reads to mimic a data set of lower coverage $2 \Lambda$, for which we test the calling of the amplified allele by HATS versus the naive method. Call accuracy for $2 \Lambda$ is reported as the fraction of correct calls across 100 such subsampling trials.

Performance is demonstrated in a glioblastoma tumor sample (TCGA-06-0877) of European descent, with whole genome sequence, array CGH, and SNP array data obtained from The Cancer Genome Atlas (Network 2008) (see Methods: Input data specifications). Chromosomes 2, 7, 12, 19, and 20 of this sample are called by array $\mathrm{CGH}$ as possessing a chromosome-wide average copy number of 2.6, suggesting a tumor copy number of 3 , with up to $40 \%$ of sample cells being nontumor-related cells of copy number 2. We considered a specific CNA region at chr19:2.18$2.54 \mathrm{Mb}$ that is reported to possess a local average coverage of $2 \bar{\Lambda}=$ 33.7 and a local average copy number of $C_{a}=3.18$, consistent with a local copy number of 4 in the $60 \%$ of sample cells that are tumor cells. Figure 3 presents call accuracy for this region across various levels of coverage $2 \Lambda$, with the gold-standard amplified alleles called by the naive method on the tumor sequence data itself. As expected, both naive calling and HATS perform well when $2 \Lambda$ is high. As $2 \Lambda$ decreases, the performance gap between HATS and the

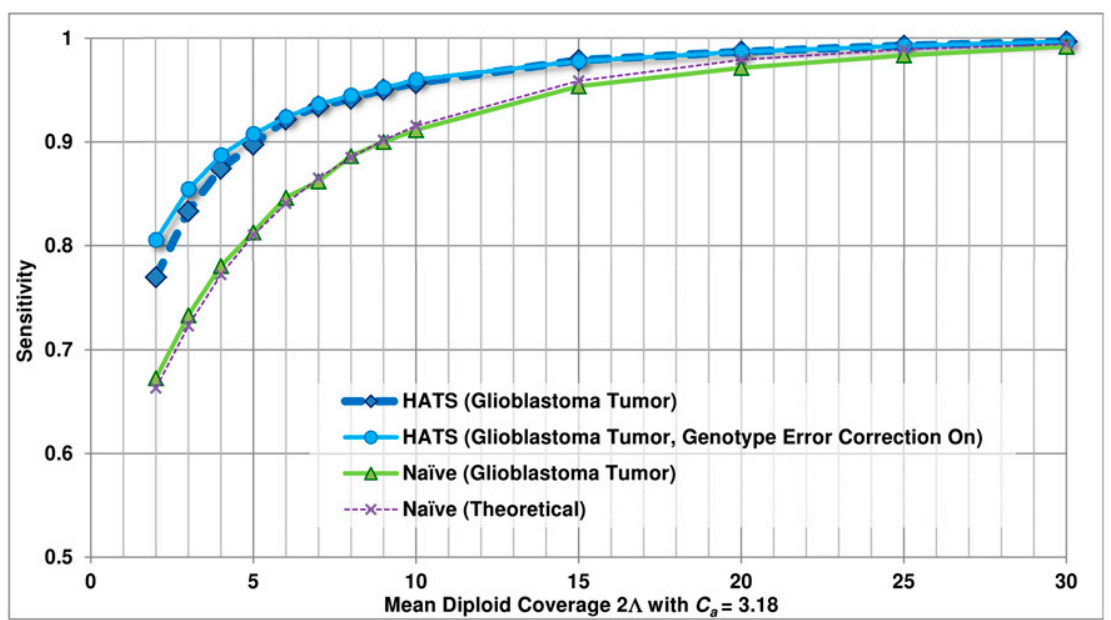

Figure 3. Empirical sensitivity of HATS and the naive model, TCGA glioblastoma sample (TCGA-060877), Chr 19. This figure displays the sensitivity results for HATS and the naive method on an amplified region (chromosome 19: 2,181,615-2,541,253) in a glioblastoma patient (TCGA-06-0877) obtained from TCGA with a local copy number of 3.18. The naive theoretical curve is included for comparison purposes. The gold-standard amplified alleles were obtained by analyzing the region with the naive method using high coverage $(33.7 \times)$ read counts, as simulations for a copy number of 3 indicated high sensitivity for the naive method at high coverage levels. The read counts were randomly down-sampled to result in varying coverage levels as displayed on the $x$-axis (with 100 trials of down-sampling performed per coverage level). The down-sampled read counts were passed to both HATS and the naive method. The reported amplified alleles were compared with the gold standard to indicate sensitivity. Note that for higher coverage levels, the performance of both HATS and the naive method is strong, which is expected as this was observed in the simulations. As coverage decreases, HATS maintains a marked performance improvement over the naive method. Tumor alignment files and copy number data for this patient were obtained from TCGA (see Methods: Input data specifications). naive method widens before shrinking slightly at low coverage. Utilizing the Genotype Error Correction, however, increases the performance. The breakdown of sensitivities per read count ob-

a site at $2 \Lambda$ is depicted in Supplemental Figure S8.

(he gold-standard amplified alleles called from the SNP array data for same sample. SNP arrays naturally possess a lower density of to only array sites forces it to ignore sites in the training data not typed on the array, resulting in weaker LD structure gleaned from the training data. Despite this, HATS retains its performance gain over the naive curve, as seen in Supplemental Figure S9. Moreover, only three heterozygous sites comprise the gold standard after quality control filtering in this case; HATS performed better than

naive method even with such few sites. contamination). We discuss findings in Supplemental Results, with HATS' markedly superior sensitivity illustrated in Supplethat HATS outperforms the naive model in real tumor data, even when sites are few and especially when coverage is reduced or

\section{Discussion}

During recent years, algorithms have been developed for SNP array platforms to determine somatic allele-specific copy numbers of pNes. Such data indicates CNAs and enables one to pinpoint potential disease-associated variants and, by virtue, haplotypes within the wide span of these regions. However, the drawbacks of these algorithms are the suboptimal resolution of the platform, and more importantly, issues with call fidelity: Amplified regions render these algorithms prone to incorrect genotype calls in tumor tissue. As NGS technologies offer nucleotide level resolution while avoiding SNP array issues that affect amplified call fidelity, we aimed to develop a novel method that could determine the amplified alleles, and thus, haplotypes in such data. To our knowledge, no other methods exist that call somatically amplified alleles and haplotypes in NGS data.

Determination of haplotypes is equivalent to locally phasing the tumor data using read counts from the tumor sample and haplotype frequencies from training data. Only one chromosome is assumed to be amplified along a homologous region. At its core, HATS builds an HMM, using allele-specific read counts as emissions and training haplotypes to model transitions. Usage of the training data is motivated by the notion that the haplotype constructed from the amplified alleles (called within an amplicon) should partially reflect a mosaic of existing haplotypes within the same population. The training haplotypes enable 
HATS to utilize linkage information from multiple sites, thus helping to improve power over that of the naive method. Within an amplicon $a$ called a priori, HATS reports the amplified allele at each site that is polymorphic either in the sample or the training data, including those sites harboring rare variants or somatic mutations. We note that HATS is also able to handle hemizygous deletion mixtures. It can identify the deleted alleles or haplotypes that are otherwise difficult to identify as deleted (due to traces of those alleles coming from contaminating stromal cells).

The assumption of one chromosome being amplified is central to HATS. While evidence of the prevalence of monoallelic amplification has been found in previous studies (LaFramboise et al. 2005), along with recent studies recounting most amplification events to be low gain (Network 2011), we acknowledge that the extent to which one or both alleles is amplified still remains an open question. HATS in its current form may produce switch errors in phasing if applied on regions with both chromosomes amplified. However, the method can be extended by adding extra states representing candidate double amplifications. Such modifications would also help HATS toward phasing germline CNV regions. Currently, HATS is equipped to locally phase germline heterozygous CNVs of copy number 3 should those regions map uniquely to the reference genome. The caveat is that samples copy-neutral for those regions would be required to calculate any allele-specific biases. Another potential extension involves incorporating pairedend information, though the practical gain from this would need to be assessed, as the mate pair distance is typically smaller than the distance between polymorphic sites.

It should be noted that the constructed amplified haplotype may differ from the true tumor haplotype with respect to the order of sites. Genomic rearrangements in tumor DNA disrupt the local cellular copy of inherited germline sequence, potentially resulting in a somatic haplotype that differs from the corresponding germline haplotype. However, rearrangement information from tumor DNA may be lost during sequencing, as single reads are mapped to the reference sequence. As such, the amplified tumor haplotype as called by HATS would reflect the order of sites in the reference genome (and the inherited germline sequence) rather than that of the rearranged tumor haplotype. A similar limitation of our approach is the potential presence of mitotic recombination. This would imply different phasing for tumor versus normal data but would typically be limited to a small number of events (Cavenee et al. 1983; Paques and Haber 1999; Barbera and Petes 2006; LaFave and Sekelsky 2009).

The design philosophy of HATS envisions the algorithm as a tool in a workflow of algorithms for studying tumors, helping to open the door for allele-specific downstream analysis. At the same time, it is reliant on upstream data; namely, the a priori calling of the amplicon. This is done intentionally, as modularity is a fundamental design principle in building complex pipelines, and it guides us here as well. Furthermore, CNA and CNV calling has been studied extensively and has matured over the years to the point that it has become integral to existing pipelines (Network $2008,2011)$. However, upstream errors can occur, some of which are safeguarded by HATS. For example, small errors in input amplicon copy number have little effect on HATS, which can naively validate copy number using tumor and matched normal read counts. The effect is further reduced due to the power HATS leverages from the training data. Other errors include amplicons that are a result of platform-specific biases (e.g., PCR bias). These can be caught by HATS' bias correction if they are allele-specific and appear in the matched normals as well. In addition, the power from the training data can help reduce the effect of spurious PCR biases that may occur within an amplicon span. The naive method, on the other hand, would be much more vulnerable to this. Other errors include inexact a priori amplicon breakpoint prediction, which could especially occur with the low resolution of arrays. Such errors may result in HATS calling an allele as amplified even if the site lies outside the amplicon in truth, or sites being ignored by HATS if, in truth, they lie within the amplicon boundary. However, some of these effects are mitigated when studying multiple tumors downstream. Amplicons due to artifact in one sample will likely not recur over multiple tumors and may thus be identified as unique or erroneous. Similarly, testing for recurrence will tease out amplicons under selection versus passenger amplicons that occur randomly and propagate due to duplication mechanisms or genomic instability during tumor evolution.

A possible alternative to HATS entails first computationally phasing the matched normal sequence (and, by virtue, the tumor sequence), ignoring valuable read count information during phasing. One might then assess tumor read counts at several sites within an amplicon to determine the particular phased haplotype targeted for amplification. We tested this approach on four regions from the glioblastoma patient (TCGA-06-0877), three of which lie on chromosome 2 and the last on chromosome 19 (see Supplemental Table S1). Only sites common to both the sequence data and SNP array were considered. The gold standard amplified alleles were determined using array calls. HATS performed with equal or greater accuracy on all four regions, while inaccuracies in phasingfirst indicated switch error. These results support HATS' relevance to amplified allelic calling. Another advantage that HATS possesses over phasing-first is the ability to make calls on rare variants or somatic mutations using read depth information. Phasing algorithms, which also rely on germline training data, seem underpowered to do this. In addition, HATS may recover heterozygous calls at low coverage via its Genotype Error Correction; performing the same with a germline phasing algorithm for sequence data would require extra preprocessing overhead for the end user. Furthermore, several phasing algorithms assume HardyWeinberg equilibrium in the test data, which may not always be the case due to the presence of risk alleles (Marchini et al. 2006). Thus, phasing the tumor via the matched normal may not always lead to accurate results. On the contrary, as the constructed tumor haplotype would reflect the genome order of the reference sequence and matched normal as mentioned above, HATS could potentially be used to phase the germline alleles in the matched normal sample based on the tumor data.

One potential future avenue involves examining haplotypes within recurrent CNA regions across tumor samples to reveal such genes. This was performed previously on SNP arrays at the single SNP level (Dewal et al. 2010; LaFramboise et al. 2010) and can now be extended to next generation sequencing data. HATS' reporting of the amplified allele may serve as a first step in this downstream analysis possibility. One benefit is that sites that were not typed on SNP arrays may be revealed to be selectively amplified across tumors in sequence data. In addition, the improved call fidelity as compared to amplified allelic calls on SNP arrays may lead to more robust results.

More generally, the HATS algorithm is designed to call allelic imbalances (AIs) at genomic sites within a sample. AIs can provide important information in multiple scenarios. For example, AI in transcripts has been detected by RNA sequencing. Transcripts demonstrating this phenomenon have genetic or epigenetic influences driving this difference (Zhang et al. 2009; Heap et al. 2010; 
Tuch et al. 2010). Chromatin immunoprecipitation followed by high-throughput sequencing, ChIP-seq, can also reveal AI at heterozygous sites. In this context, allelic imbalance can reflect differential binding specificity of a particular DNA-protein interaction, thereby possibly highlighting functional variants.

In the pursuit of detecting causal or associated variants, it aids the cancer community to integrate somatic and germline DNA changes. We present a method that helps move toward this end in next generation tumor sequence data. The strong evaluation of HATS provides us confidence to offer the community this powerful local phasing method. We hope that cancer researchers will find it beneficial toward discovering variants and potential oncogenes.

\section{Methods}

\section{Naive model parameters and input data specifications}

We denote the read count for allele $x \in\{\mathbf{0}, \mathbf{1}\}$ at a genomic site $i$ (a potential SNV site) in the tumor sample of individual $j$ by $r_{x}$. Formally, $r_{x}$ would be indexed as $r_{i, j, x}$, though indices for the site and individual are omitted for simplicity in the running text when clear from context. In the set of regions amplified in this sample, we consider each amplicon $a$ in turn and denote its boundaries by $i_{a}^{-}$and $i_{a}^{+}$. At an amplified heterozygous site $i \in\left[i_{a}^{-}, i_{a}^{+}\right]$, it is meaningful to ask which allele is amplified. A naive model for calling the amplified allele would simply choose the allele for which a greater number of reads is observed, denoted as $\arg -\max _{\boldsymbol{x}}\left\{r_{x}\right\}$. A call is avoided in case of a tie.

With a probabilistic view in mind, $r_{x}$ is a value of a random variable $\boldsymbol{R}_{\boldsymbol{x}} \sim$ Poisson $\left(\lambda_{x}\right)$, where $\lambda_{x}$ represents the site-, individual-, and allele-specific expectation for the number of tumor reads. This formulation interprets the naive call as choosing the allele for which the maximum likelihood estimate of $\lambda_{x}$ is greater. This model makes use of no input data other than $r_{x}$ and calls sites along the amplicon $a$ independently of one another. It thus serves as a suitable performance baseline against HATS.

\section{HATS model parameters}

The contribution of this manuscript involves recovering the amplified allele based on a more careful modeling of $\lambda_{x}$. Specifically, $\lambda_{0}$ and $\lambda_{1}$ are assumed to be proportional to the number of copies of the respective alleles at site $i$ in the tumor sample $j$ at hand. We denote these numbers, or tumor genotype calls, by $G_{0}$ and $G_{1}$, respectively. The total copy number $C_{a}=G_{0}+G_{1}$ at site $i$ is $>2$ in an amplicon $a$, and our task of identifying the amplified allele is tantamount to distinguishing between the cases $\left(G_{0}=C_{a}-1 ; G_{1}=1\right)$ and $\left(G_{0}=1 ; G_{1}=C_{a}-1\right)$. Furthermore, $\lambda_{x}$ is assumed to be proportional to $\Lambda$, the average haploid coverage of the sequenced tumor $j ; \Lambda$ is a sample-specific quantity that depends on the sequencing resources invested in the data for that tumor. Lastly, NGS reads are known to be often biased toward particular nucleotides (Bentley et al. 2008; McKernan et al. 2009). Therefore, a realistic and general model for $\lambda_{x}$ needs to account for such a site-specific (versus samplespecific) phenomenon. Our model then assumes this parameter is proportional to an allelic bias factor $b_{x}$ local to this site. In summary, including site- and sample-specific subscripts $(i, j)$ for completeness of the formal equation:

$$
\lambda_{i, j, x}=\Lambda_{j} \cdot G_{i, j, x} \cdot b_{i, x}
$$

Note that by modifying the Poisson mean $\Lambda$ via $b_{x}$, the model accommodates for genome-wide overdispersion in which the variance in read counts may exceed the expected variance (also $\Lambda$ ). Overdispersion in general may result from a positive correlation between events (Robinson and Smyth 2008) (e.g., overlapping reads covering a common interval) or from differing expected numbers of reads at different sites (Bentley et al. 2008; McKernan et al. 2009). Since each site may possess a unique bias $b_{x}$, the siteand allele-specific Poisson mean $\lambda_{x}$ may also be unique at each site $i$ in sample $j$. The combination of the varying $\lambda_{x}$ across sites effectively implements the negative binomial (NB) distribution genomewide. Existing methods estimate and use the NB distribution directly to model overdispersion in other types of sequencing data, such as in RNA-seq (Anders and Huber 2010; Robinson et al. 2010) and ChIP-seq (Anders and Huber 2010).

The definitions of $\lambda_{x}, G_{x}$, and $C_{a}$ easily generalize to homozygous sites (where $G_{x}=C_{a}$ for one of the alleles and $G_{x}=0$ for the other) as well as copy neutral sites $\left(C_{a}=2\right)$. Furthermore, we consider the sequencing of not only the tumor but also of matched normal samples of the corresponding individuals (denoted by operator ). Our model analyzes a tumor amplicon $a$ only if the corresponding region in the matched normal is copy-neutral, thus preventing germline $\mathrm{CNV}$ regions from confounding the analysis. Read counts $\tilde{r}_{x}$ for normal samples are analogously defined, as are the random variables: $\tilde{\boldsymbol{R}}_{\boldsymbol{x}} \sim \operatorname{Poisson}\left(\tilde{\lambda}_{x}\right)$, where $\tilde{C}_{a}=\tilde{G}_{0}+\tilde{G}_{1}=2$, and

$$
\tilde{\lambda}_{i, j, x}=\tilde{\Lambda}_{j} \cdot \tilde{G}_{i, j, x} \cdot b_{i, x} \cdot
$$

The genotype call at a site is, in fact, comprised of the haplotype calls at that site. We aim to distinguish the two haploid copies of the genome giving rise to the tumor genotype call. Within an amplicon $a$, we assume one of the haplotypes is (A)mplified while the other is $(\boldsymbol{U})$ namplified (LaFramboise et al. 2005). We formally represent these ground truth haplotypes as binary strings $q_{a}^{A}$ and $q_{a}^{U}$, respectively, each listing the alleles along its sequence. We define the complement operator for allele $x$ such that $\overline{0}=1$ and $\overline{1}=0$. At a particular site $i \in\left[i_{a}^{-}, i_{a}^{+}\right]$, we denote by $H_{x}^{y}$ the number of copy-neutral copies (0 or 1$)$ of $x$ along $q_{a}^{y}$, where $y \in\{A, U\}$; this is depicted in Figure 4A. Naturally, the tumor genotype call for an amplified allele $x$ sums the copies on both haplotype calls, with amplification of a particular haplotype call represented by multiplication with a constant $\left(C_{a}-1\right)$ :

$$
G_{i, j, x}=\left(C_{a}-1\right) H_{i, j, x}^{A}+H_{i, j, x}^{U} .
$$

The tumor genotype call for a nonamplified allele $\bar{x}$ is similar:

$$
G_{i, j, \bar{x}}=H_{i, j, \bar{x}}^{A}+H_{i, j, \bar{x}}^{U} .
$$

For the matched normal, the genotype call sums the copies of two nonamplified haplotype calls, as per the requirements of copy neutrality (see Fig. 4A):

$$
\tilde{G}_{i, j, x}=\sum \tilde{H}_{i, j, x}^{U}
$$

\section{HATS hidden Markov model}

We use an accepted model of human variation (Kimmel and Shamir 2005; Rastas et al. 2005; Eronen et al. 2006; Browning and Browning 2007; Sun et al. 2007b), presenting haplotypes $\hat{q}_{a}^{A}$ and $\hat{q}_{a}^{U}$ as the output of two respective haplotype HMMs $V^{A}$ and $V^{U}$, detailed below. We take a Cartesian product of the two haplotype HMMs to define a genotype HMM, for which the genotypes are the output. The read counts $r_{x}$ are a probabilistic function of these genotypes, as explained above. HATS entails deciphering, for each site and sample, the likelihood of states within this genotype model, and then choosing the allele most likely to have been amplified. An overview of the model is provided in Figure 4B-E.

\section{Haplotype HMM}

In detail, the haplotype HMM $V^{y}$ is a state machine with probabilistic transitions and deterministic emissions. The states and 

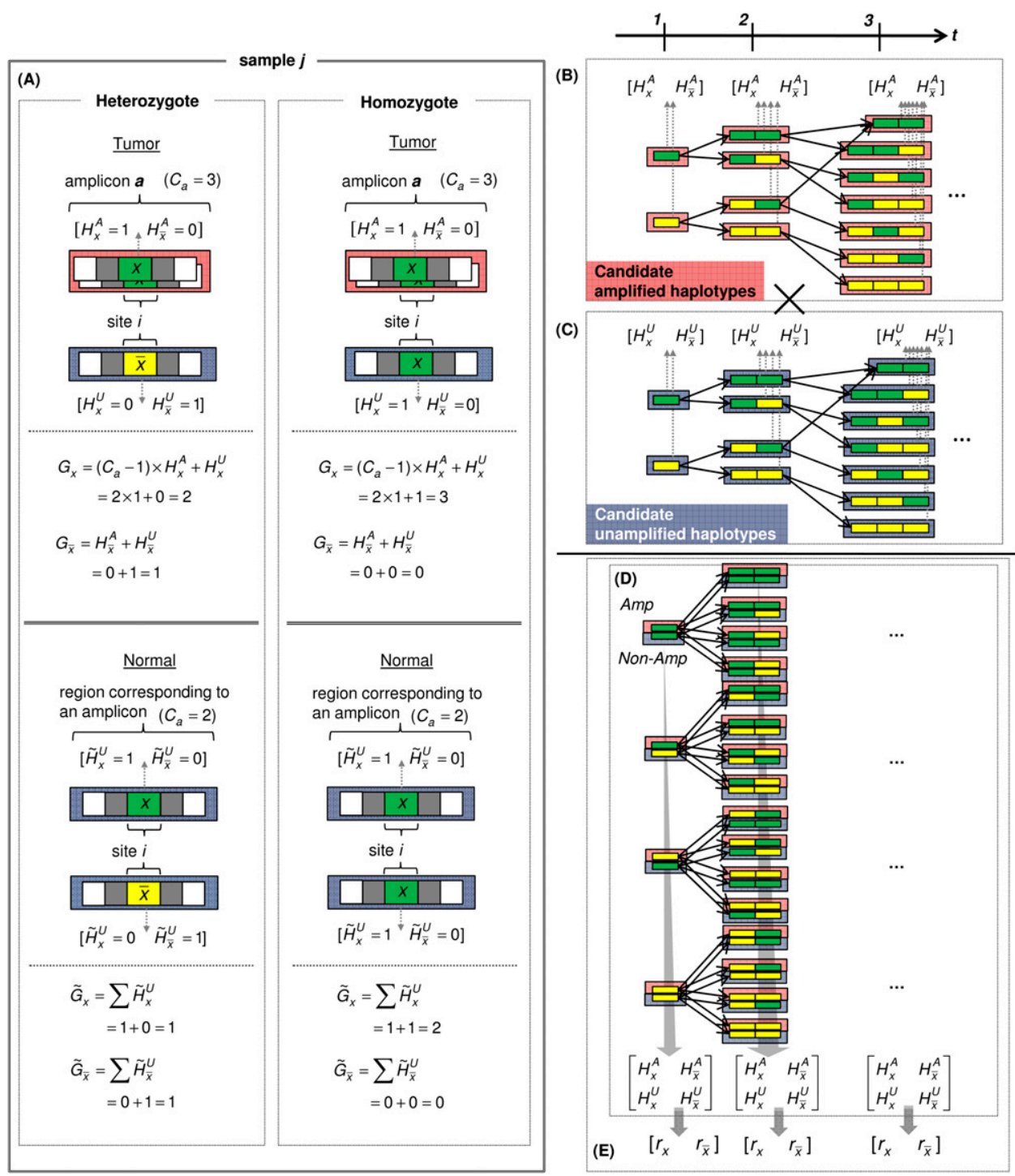

Figure 4. Parameter definition depictions, haplotype, genotype, and integrated HMM. The left half of the figure depicts the definitions of several model parameters, while the right half depicts examples of the four HMM models. (A) Model parameters are depicted for a heterozygous or homozygous site $i$ in tumor and matched normal tissues for sample $j$. For a particular genotype call within a tissue, a diagram of the call is depicted, along with the corresponding $H$ and $G$ parameters. For example, for a heterozygous call within a tumor, the amplicon $a$ (containing $i$ ) from the tumor is shown, depicting the amplified haplotype (with allele $x$ amplified) within the red frame and nonamplified haplotype (allele $\bar{x}$ ) within the blue frame. The haplotypes emit their respective $H$ values per site, followed by the definitions of the $G$ values for each allele. Both $H$ and $G$ should be additionally subscripted by $i$ and $j$ formally, but these subscripts are excluded for simplicity's sake. For the amplified haplotype in red, $H_{x}^{A}=1$ since $x$ is present (and amplified), while $H_{\bar{x}}^{A}=0$ since $\bar{x}$ is not present within that haplotype at $i$. For the nonamplified haplotype in blue, $H_{\bar{x}}^{U}=1$ since $\bar{x}$ is present (and not amplified), while $H_{x}^{U}=0$ since $x$ is not present within that haplotype at $i$. (B) Example of the haplotype HMM for the amplified haplotype. Each state contained within a red frame represents a candidate amplified haplotype. Green boxes represent $x$ while yellow boxes represent $\bar{x}$. The $H$ symbols emitted from a state correspond to the last allele in the haplotype represented by the state, as depicted by the dotted vertical arrows. The haplotype lengths generally grow from left to right due to addition of an allele at the current $t$. Note that at $t=3$, most states represent haplotypes of length 3 , with the exception of the topmost state, which represents a haplotype [green $\mid$ green] of length 2. This haplotype merges two 3-SNP haplotypes [green $\mid$ green $\mid$ green] and [yellow $\mid$ green $\mid$ green] because they both are singletons in the training data. Both states [green $\mid$ green] and [yellow $\mid$ green] at $t=2$ thus transition to state [green $\mid$ green] at $t=3$. (C) Haplotype HMM for the nonamplified haplotype. Note that it is identical to $B$ except for the differing emitted symbols. $(D)$ Genotype HMM that is the cross-product of the haplotype HMMs. For each pair of haplotypes, the amplified haplotype is depicted above the nonamplified haplotype within red and blue frames, respectively. Each pair of haplotypes inherits the $H$ values from their respective haplotype HMMs. (E) Translated HMM upon incorporating the model data. Note that while the structure remains unchanged, the emitted symbols are translated from the $H$ values to the read counts. The $r_{x}$ variables should also include subscripts $i$ and $j$ for formal correctness, which were omitted here for simplicity's sake.

transitions follow the haplotype models used by computational phasing algorithms for SNP array data (Kimmel and Shamir 2005; Rastas et al. 2005; Eronen et al. 2006; Browning and Browning 2007; Sun et al. 2007b). In a similar vein, we locally phase the tumor sequence data based on read counts in the sample data and haplotype counts in population-relevant training data.

The "time points" of $V^{y}$ consist of the set of sites $i_{1}, i_{2}, \ldots, i_{M}$ located within the amplicon $a$ coordinates that are polymorphic

\section{Genome Research} www.genome.org 
(i.e., include a nonreference allele call) in the germline of the training data. Sites that are monomorphic in the training data do not provide information regarding the amplified allele and need not be analyzed here. Each time point $t$ in $V^{y}$ maps to a genomic site $i_{t}$ and is associated with a set of HMM states $S_{t}^{y}$. Each $s \in S_{t}^{y}$ is labeled by a binary string $h_{s}$, which represents a candidate local haplotype for polymorphic sites $i_{t-l(s)+1}, \ldots, i_{t}$, where $l(s)=\left|h_{s}\right|$. State $s$ emits $H_{x}^{y}$ values deterministically depending on $h_{s}[l(s)]$, the last symbol in $h_{s}: H_{x}^{y}=1$ for $x=h_{s}[l(s)]$ and $H_{\bar{\chi}}^{y}=0$ for the complement allele. For every $s \in S_{t}^{y}$ in which $t>1$, there exists at least one $\hat{s} \in S_{t-1}^{y}$ that transitions to $s$ such that they are consistent: The prefix of $h_{s}$ of length $(l(s)-1)$, denoted as $h_{s}^{*}$, is a suffix of $h_{\hat{s}}$. This relationship can be visualized in Figure 4B,C. Transitions are allowed only between consistent states.

Any of the multiple methods for learning a haplotype HMM from training data may be employed to choose lengths of state labels $h_{s}$ and transition probabilities between a pair of consistent states. We implemented a simple such method, which relies on counts of $h_{s}$ in the training (D)ata ending at $t$-denoted by $D_{t}\left(h_{s}\right)$. The concept behind this is that, at a given locus, a germline haplotype of a tumor sample is assumed to be identical to one of the training haplotypes, randomly switching the training sample that is locally identical to the tumor haplotype along the genome. This concept is a standard in germline genetics (Stephens et al. 2001; Scheet and Stephens 2006; Browning and Browning 2007). The candidate haplotype label of $s$ is thus tested for such a local matching. We enforce $h_{s}$ labels to be as long as possible while maintaining $D_{t}\left(h_{s}\right) \geq \min (2, l(s))$. This constraint prevents creating labels that are void in $D$ or labels of length $>1$ that are singletons in $D$ (to prevent overfitting), while, on the other hand, allowing labels of length 1 that represent rare or low frequency single nucleotide variants in $D$. The length of state labels thus trades off accuracy of training vs. overfitting.

The transition probability between consistent states $\hat{s}$ and $s$ is set as a Dirichlet prior based on $D_{t}\left(h_{s}\right)$ :

$$
\begin{aligned}
\operatorname{Pr}\left(h_{s} \mid h_{s}^{*}\right) & =\frac{D_{t}\left(h_{s}\right)}{D_{t-1}\left(h_{s}^{*}\right)}, \quad \text { if } l(s)>1 \\
& =\text { freq }\left(h_{s}, D, t\right) \text {, if } l(s)=1 \text { and } D_{t}\left(h_{s}\right) \geq 1 .
\end{aligned}
$$

The function freq() represents the haplotype frequency of $h_{s}$ in $D$ ending at $t$. Recall that we never encounter the case in which $\left|h_{s}\right|=1$ and $D_{t}\left(h_{s}\right)=0$, as we ignore sites monomorphic in $D$. Such sites that are polymorphic only in the test sample data (rare variants or somatic mutations) are handled external to the HMM and are described in the section Enhancements and Optimizations below.

\section{Genotype HMM}

We construct a genotype HMM $V$ by cross-multiplying $V^{A}$ and $V^{U}$ (see Fig. 4D). The $M$ time points remain unchanged. At a given $t$, the set of states is the Cartesian product $S_{t}=S_{t}^{A} \times S_{t}^{U}$. Thus, for each $s=\left(s^{A}, s^{U}\right) \in S_{t}, s^{A} \in S_{t}^{A}$ and $s^{U} \in S_{t}^{U}$. State $s$ thus inherits its labels, denoted as $\left(h_{s}^{A}, h_{s}^{U}\right)$, from its component states. Likewise, $s$ inherits the $H_{x}^{y}$ values (over all $y$ and $x$ ), which it emits deterministically. The transition probability from $\hat{s}=\left(\hat{s}^{A}, \hat{s}^{U}\right) \in S_{t-1}$ to $s$ is the product of the transition probabilities to the component states of $s$ :

Transition Probability of $\hat{s}$ to $s=\operatorname{Pr}\left(h_{s}^{A} \mid h_{s}^{A *}\right) \times \operatorname{Pr}\left(h_{s}^{U} \mid h_{s}^{U *}\right)$.

\section{Integrated HMM}

Our model for the read data is a new HMM $V^{+}$that is a replica of $V$, adding stochastic emissions on top of it (see Fig. 4E). For each state $s \in S_{t}^{+}$, the emitted symbols are, instead, the read counts $r_{x}$ for each allele $x$. Each $s$ is still labeled with $\left(h_{s}^{A}, h_{s}^{U}\right)$ and inherently represents the $H_{x}^{y}$ values (over all $y$ and $x$ ). Assuming $C_{a}$ is known beforehand and that $b_{x}$ is calculated, parameters $G_{x}$ and $\lambda_{x}$ can be calculated from $H_{x}^{y}$ (over all $y$ and $x$ ). The emission probability for $s$ is the probability of observing $r_{x}$, assuming an underlying Poisson distribution for the read counts at site $i_{t}$ with mean $\lambda_{x}$. It is calculated on the Poisson distribution as

$$
\text { Emission Probability at } s=\prod_{x=0}^{1} \operatorname{Pr}\left(r_{i, j, x} ; \lambda_{i, j, x}\right) \text {. }
$$

\section{HMM deciphering algorithm to call the amplified allele}

The ultimate goal of the HMM is to decipher which allele $x$ is amplified at $t$. Toward this end, we apply the forward-backward algorithm on $V^{+}$, resulting in a probability $P(s)$ for each state in $V^{+}$. Let $S_{t, x}^{+} \subseteq S_{t}^{+}$represent those states whose amplified haplotype labels $h_{s}^{A}$ end in $x$, or more formally, whose $h_{s}^{A}$ labels possess $x$ as the last symbol. HATS computes the total likelihood for each allele $x$ by summing over the relevant $P(s)$ values:

$$
L_{t}(x)=\sum_{s \in S_{t, x}^{+}} P(s) .
$$

HATS finally reports the most likely such $x$ to have been amplified at $t$ via: $\arg -\max _{x}\left\{L_{t}(x)\right\}$. In the case of a tie, HATS designates $\varnothing$ as the amplified allele at $t$ to reflect this ambiguity.

\section{Input data specifications}

HATS jointly considers input regarding $n$ tumor and matched normal samples $(1 \leq j \leq n)$. Specifically, input data corresponding to a particular sample $j$ includes the input data from the naive model, in addition to the following:

(1) $C_{a}$ (>2, obtained by preprocessing with a copy number-calling algorithm (Chiang et al. 2009) or typing the sample on another platform, e.g., array CGH);

(2) $\Lambda$ and $\tilde{\Lambda}$ (calculable beforehand);

(3) $\tilde{r}_{x}$ and $\tilde{G}_{x}$ for all $i$ in each of the $n$ samples that are copy-neutral at $i$, with the constraint that $i$ resides in amplicon $a$ in sample $j$; and

(4) genotypes from the tumor of individual $j$ that do not take copy number into account.

The latter two are obtainable via a genotype caller component from an alignment algorithm (Li et al. 2009). The third input specification-read counts from each $i$ within copy-neutral regions in tumor and matched normal samples, such that $i$ resides in an amplicon in at least one of the $n$ input tumor samples-allows for calculation of the site-specific bias factor $b_{x}$. Estimation of $b_{x}$ and the effects of the availability of matched normal data on this estimation are discussed in Supplemental Methods. This tumor and matched normal input data can be derived from aligned sequence data obtainable from any sequencing project or initiative. The tumor and matched normal data we used in Results was downloaded from TCGA. The respective URL and relevant $\mathrm{dbGaP}$ accession number are http://tcga-data.nci.nih.gov/tcga/, phs000178.v4.p4.

Lastly, HATS takes advantage of linkage information across multiple sites to call the amplified alleles, whereas the naive model ignores LD by examining each site individually. Toward this end, HATS uses training data (denoted above as $D$ ) for human germline variation, which entails phased haplotype sequence data on $d$ unrelated individuals that are independently sampled from the same population as the $n$ cancer patients whose tumors are to be analyzed. The training population can be determined as a preprocessing step using ancestry informative markers in the tumor samples. 
The training data itself can be obtained, for example, from the 1000 Genomes Project (The 1000 Genomes Project Consortium 2010).

\section{Enhancements and optimizations}

Tumor samples may include sites housing somatic mutations or variants that are unique to the samples and missing in the training data (e.g., singletons). HATS utilizes read depth, along with bias determination, to call the amplified allele at each such site. In addition, HATS possesses the ability to potentially recover an allele missed due to low coverage (Genotype Error Correction); this is integrated with a mechanism to utilize tumor genotypes to prune the explosive growth of states. Details on these enhancements are provided in Supplemental Methods.

\section{Data access}

The HATS source code, as well as instructions to build and run the software, is available at (http://tumorhats.sourceforge.net/).

\section{Acknowledgments}

This work was supported by the National Institutes of Health and National Library of Medicine (5T15 LM007079-14 to the Medical Informatics Research Training Program, Department of Biomedical Informatics, Columbia University); and the National Institutes of Health (1 R01 CA131341-01A1). The results published here are, in part, based upon data generated by The Cancer Genome Atlas pilot project established by the NCI and NHGRI. Information about TCGA, the investigators, and institutions that constitute the TCGA research network can be found at http://cancergenome.nih.gov. The results here are also, in part, based upon data generated by the 1000 Genomes Project. Information about the 1000 Genomes Project, along with participating investigators and institutions, can be found at http://www.1000genomes.org.

\section{References}

The 1000 Genomes Project Consortium. 2010. A map of human genome variation from population-scale sequencing. Nature 467: 1061-1073.

Ahmed S, Thomas G, Ghoussaini M, Healey CS, Humphreys MK, Platte R, Morrison J, Maranian M, Pooley KA, Luben R, et al. 2009. Newly discovered breast cancer susceptibility loci on 3p24 and 17q23.2. Nat Genet 41: 585-590.

Alkan C, Kidd JM, Marques-Bonet T, Aksay G, Antonacci F, Hormozdiari F, Kitzman JO, Baker C, Malig M, Mutlu O, et al. 2009. Personalized copy number and segmental duplication maps using next-generation sequencing. Nat Genet 41: 1061-1067.

Amos CI, Wu X, Broderick P, Gorlov IP, Gu J, Eisen T, Dong Q, Zhang Q, Gu $\mathrm{X}$, Vijayakrishnan J, et al. 2008. Genome-wide association scan of tag SNPs identifies a susceptibility locus for lung cancer at 15q25.1. Nat Genet 40: 616-622.

Amundadottir LT, Sulem P, Gudmundsson J, Helgason A, Baker A, Agnarsson BA, Sigurdsson A, Benediktsdottir KR, Cazier JB, Sainz J, et al. 2006. A common variant associated with prostate cancer in European and African populations. Nat Genet 38: 652-658.

Anders S, Huber W. 2010. Differential expression analysis for sequence count data. Genome Biol 11: R106. doi: 10.1186/gb-2010-11-10-r106

Barbera MA, Petes TD. 2006. Selection and analysis of spontaneous reciprocal mitotic cross-overs in Saccharomyces cerevisiae. Proc Natl Acad Sci 103: $12819-12824$.

Bentley DR, Balasubramanian S, Swerdlow HP, Smith GP, Milton J, Brown CG, Hall KP, Evers DJ, Barnes CL, Bignell HR, et al. 2008. Accurate whole human genome sequencing using reversible terminator chemistry. Nature 456: 53-59.

Bentz M, Plesch A, Stilgenbauer S, Dohner H, Lichter P. 1998. Minimal sizes of deletions detected by comparative genomic hybridization. Genes Chromosomes Cancer 21: 172-175.

Browning SR, Browning BL. 2007. Rapid and accurate haplotype phasing and missing-data inference for whole-genome association studies by use of localized haplotype clustering. Am J Hum Genet 81: 1084-1097.
Cavenee WK, Dryja TP, Phillips RA, Benedict WF, Godbout R, Gallie BL, Murphree AL, Strong LC, White RL. 1983. Expression of recessive alleles by chromosomal mechanisms in retinoblastoma. Nature 305: 779784.

Chen K, Wallis JW, McLellan MD, Larson DE, Kalicki JM, Pohl CS, McGrath SD, Wendl MC, Zhang Q, Locke DP, et al. 2009. BreakDancer: An algorithm for high-resolution mapping of genomic structural variation. Nat Methods 6: 677-681.

Cher ML, Bova GS, Moore DH, Small EJ, Carroll PR, Pin SS, Epstein JI, Isaacs WB, Jensen RH. 1996. Genetic alterations in untreated metastases and androgen-independent prostate cancer detected by comparative genomic hybridization and allelotyping. Cancer Res 56: 3091-3102.

Chiang DY, Getz G, Jaffe DB, O'Kelly MJ, Zhao X, Carter SL, Russ C, Nusbaum C, Meyerson M, Lander ES. 2009. High-resolution mapping of copy-number alterations with massively parallel sequencing. Nat Methods 6: 99-103.

Chung CC, Magalhaes WC, Gonzalez-Bosquet J, Chanock SJ. 2010. Genome-wide association studies in cancer-Current and future directions. Carcinogenesis 31: 111-120.

Dalca AV, Brudno M. 2010. Genome variation discovery with highthroughput sequencing data. Brief Bioinform 11: 3-14.

de Koning JP, Wakabayashi Y, Nagase H, Mao JH, Balmain A. 2007. Convergence of congenic mapping and allele-specific alterations in tumors for the resolution of the Skts1 skin tumor susceptibility locus. Oncogene 26: 4171-4178.

Dewal N, Freedman ML, LaFramboise T, Pe'er I. 2010. Power to detect selective allelic amplification in genome-wide scans of tumor data. Bioinformatics 26: 518-528.

Easton DF, Eeles RA. 2008. Genome-wide association studies in cancer. Hum Mol Genet 17: R109-R115.

Eronen L, Geerts F, Toivonen H. 2006. HaploRec: Efficient and accurate large-scale reconstruction of haplotypes. BMC Bioinformatics 7: 542. doi: 10.1186/1471-2105-7-542.

Ewart-Toland A, Briassouli P, de Koning JP, Mao JH, Yuan J, Chan F, MacCarthy-Morrogh L, Ponder BA, Nagase H, Burn J, et al. 2003. Identification of Stk6/STK15 as a candidate low-penetrance tumorsusceptibility gene in mouse and human. Nat Genet 34: 403-412.

Fletcher O, Johnson N, Gibson L, Coupland B, Fraser A, Leonard A, dos Santos Silva I, Ashworth A, Houlston R, Peto J. 2008. Association of genetic variants at 8q24 with breast cancer risk. Cancer Epidemiol Biomarkers Prev 17: 702-705.

Freedman ML, Haiman CA, Patterson N, McDonald GJ, Tandon A, Waliszewska A, Penney K, Steen RG, Ardlie K, John EM, et al. 2006. Admixture mapping identifies 8q24 as a prostate cancer risk locus in African-American men. Proc Natl Acad Sci 103: 14068-14073.

Gharizadeh B, Nordstrom T, Ahmadian A, Ronaghi M, Nyren P. 2002. Long-read pyrosequencing using pure 2'-deoxyadenosine-5'-O'(1-thiotriphosphate) Sp-isomer. Anal Biochem 301: 82-90.

Hall JM, Lee MK, Newman B, Morrow JE, Anderson LA, Huey B, King MC. 1990. Linkage of early-onset familial breast cancer to chromosome 17q21. Science 250: 1684-1689.

Hartl DL, Clark AG. 2007. Principles of population genetics. Sinauer Associates, Sunderland, MA.

Heap GA, Yang JH, Downes K, Healy BC, Hunt KA, Bockett N, Franke L, Dubois PC, Mein CA, Dobson RJ, et al. 2010. Genome-wide analysis of allelic expression imbalance in human primary cells by highthroughput transcriptome resequencing. Hum Mol Genet 19: 122134.

Hienonen T, Salovaara R, Mecklin JP, Jarvinen H, Karhu A, Aaltonen LA. 2006. Preferential amplification of AURKA 91A (Ile31) in familial colorectal cancers. Int J Cancer 118: 505-508.

Hindorff LA, Sethupathy P, Junkins HA, Ramos EM, Mehta JP, Collins FS, Manolio TA. 2009. Potential etiologic and functional implications of genome-wide association loci for human diseases and traits. Proc Natl Acad Sci 106: 9362-9367.

Hormozdiari F, Alkan C, Eichler EE, Sahinalp SC. 2009. Combinatorial algorithms for structural variation detection in high-throughput sequenced genomes. Genome Res 19: 1270-1278.

Hung RJ, McKay JD, Gaborieau V, Boffetta P, Hashibe M, Zaridze D, Mukeria A, Szeszenia-Dabrowska N, Lissowska J, Rudnai P, et al. 2008. A susceptibility locus for lung cancer maps to nicotinic acetylcholine receptor subunit genes on 15q25. Nature 452: 633-637.

The International HapMap Consortium. 2005. A haplotype map of the human genome. Nature 437: 1299-1320.

Ishkanian AS, Malloff CA, Watson SK, DeLeeuw RJ, Chi B, Coe BP, Snijders A, Albertson DG, Pinkel D, Marra MA, et al. 2004. A tiling resolution DNA microarray with complete coverage of the human genome. Nat Genet 36: 299-303.

Jones AV, Chase A, Silver RT, Oscier D, Zoi K, Wang YL, Cario H, Pahl HL, Collins A, Reiter A, et al. 2009. JAK2 haplotype is a major risk factor for

\section{Genome Research}


the development of myeloproliferative neoplasms. Nat Genet 41: 446449.

Joos S, Bergerheim US, Pan Y, Matsuyama H, Bentz M, du Manoir S, Lichter P. 1995. Mapping of chromosomal gains and losses in prostate cancer by comparative genomic hybridization. Genes Chromosomes Cancer 14: 267-276.

Kallioniemi A, Kallioniemi OP, Sudar D, Rutovitz D, Gray JW, Waldman F, Pinkel D. 1992. Comparative genomic hybridization for molecular cytogenetic analysis of solid tumors. Science 258: 818-821.

Kilpivaara O, Mukherjee S, Schram AM, Wadleigh M, Mullally A, Ebert BL, Bass A, Marubayashi S, Heguy A, Garcia-Manero G, et al. 2009. A germline JAK2 SNP is associated with predisposition to the development of JAK2(V617F)-positive myeloproliferative neoplasms. Nat Genet 41: $455-459$.

Kimmel G, Shamir R. 2005. A block-free hidden Markov model for genotypes and its application to disease association. J Comput Biol 12: 1243-1260.

Komura D, Shen F, Ishikawa S, Fitch KR, Chen W, Zhang J, Liu G, Ihara S, Nakamura H, Hurles ME, et al. 2006. Genome-wide detection of human copy number variations using high-density DNA oligonucleotide arrays. Genome Res 16: 1575-1584.

Korbel JO, Urban AE, Affourtit JP, Godwin B, Grubert F, Simons JF, Kim PM Palejev D, Carriero NJ, Du L, et al. 2007. Paired-end mapping reveals extensive structural variation in the human genome. Science 318: 420426.

Korn WM, Yasutake T, Kuo WL, Warren RS, Collins C, Tomita M, Gray J, Waldman FM. 1999. Chromosome arm 20q gains and other genomic alterations in colorectal cancer metastatic to liver, as analyzed by comparative genomic hybridization and fluorescence in situ hybridization. Genes Chromosomes Cancer 25: 82-90.

Korn JM, Kuruvilla FG, McCarroll SA, Wysoker A, Nemesh J, Cawley S, Hubbell E, Veitch J, Collins PJ, Darvishi K, et al. 2008. Integrated genotype calling and association analysis of SNPs, common copy number polymorphisms and rare CNVs. Nat Genet 40: 1253-1260.

LaFave MC, Sekelsky J. 2009. Mitotic recombination: Why? when? how? where? PLoS Genet 5: e1000411. doi: 10.1371/journal.pgen.1000411.

LaFramboise T, Weir BA, Zhao X, Beroukhim R, Li C, Harrington D, Sellers WR, Meyerson M. 2005. Allele-specific amplification in cancer revealed by SNP array analysis. PLoS Comput Biol 1: e65. doi: 10.1371/journal. pcbi.0010065.

Laframboise T, Harrington D, Weir BA. 2007. PLASQ: A generalized linear model-based procedure to determine allelic dosage in cancer cells from SNP array data. Biostatistics 8: 323-336.

LaFramboise T, Dewal N, Wilkins K, Pe'er I, Freedman ML. 2010. Allelic selection of amplicons in glioblastoma revealed by combining somatic and germline analysis. PLoS Genet 6: e1001086. doi: 10.1371/journal. pgen.1001086.

Langmead B, Trapnell C, Pop M, Salzberg SL. 2009. Ultrafast and memoryefficient alignment of short DNA sequences to the human genome. Genome Biol 10: R25. doi: 10.1186/gb-2009-10-3-r25.

Le Marchand L. 2009. Genome-wide association studies and colorectal cancer. Surg Oncol Clin N Am 18: 663-668.

Lee S, Hormozdiari F, Alkan C, Brudno M. 2009. MoDIL: Detecting small indels from clone-end sequencing with mixtures of distributions. Nat Methods 6: 473-474.

Li H, Durbin R. 2009. Fast and accurate short read alignment with BurrowsWheeler transform. Bioinformatics 25: 1754-1760.

Li H, Ruan J, Durbin R. 2008a. Mapping short DNA sequencing reads and calling variants using mapping quality scores. Genome Res 18: 18511858.

Li R, Li Y, Kristiansen K, Wang J. 2008b. SOAP: Short oligonucleotide alignment program. Bioinformatics 24: 713-714.

Li H, Handsaker B, Wysoker A, Fennell T, Ruan J, Homer N, Marth G, Abecasis G, Durbin R. 2009. The sequence alignment/map format and SAMtools. Bioinformatics 25: 2078-2079.

Lin M, Wei LJ, Sellers WR, Lieberfarb M, Wong WH, Li C. 2004. dChipSNP: Significance curve and clustering of SNP-array-based loss-ofheterozygosity data. Bioinformatics 20: $1233-1240$.

Marchini J, Cutler D, Patterson N, Stephens M, Eskin E, Halperin E, Lin S, Qin ZS, Munro HM, Abecasis GR, et al. 2006. A comparison of phasing algorithms for trios and unrelated individuals. Am J Hum Genet 78: 437450.

McCarthy MI, Abecasis GR, Cardon LR, Goldstein DB, Little J, Ioannidis JP, Hirschhorn JN. 2008. Genome-wide association studies for complex traits: Consensus, uncertainty, and challenges. Nat Rev Genet 9: 356369.

McKernan KJ, Peckham HE, Costa GL, McLaughlin SF, Fu Y, Tsung EF, Clouser CR, Duncan C, Ichikawa JK, Lee CC, et al. 2009. Sequence and structural variation in a human genome uncovered by short-read, massively parallel ligation sequencing using two-base encoding. Genome Res 19: 1527-1541.
Medvedev P, Fiume M, Dzamba M, Smith T, Brudno M. 2010. Detecting copy number variation with mated short reads. Genome Res 20: 1613-1622.

Miki Y, Swensen J, Shattuck-Eidens D, Futreal PA, Harshman K, Tavtigian S, Liu Q, Cochran C, Bennett LM, Ding W, et al. 1994. A strong candidate for the breast and ovarian cancer susceptibility gene BRCA1. Science 266: 66-71.

Nagase H, Mao JH, Balmain A. 2003. Allele-specific Hras mutations and genetic alterations at tumor susceptibility loci in skin carcinomas from interspecific hybrid mice. Cancer Res 63: 4849-4853.

Nannya Y, Sanada M, Nakazaki K, Hosoya N, Wang L, Hangaishi A, Kurokawa M, Chiba S, Bailey DK, Kennedy GC, et al. 2005. A robust algorithm for copy number detection using high-density oligonucleotide single nucleotide polymorphism genotyping arrays. Cancer Res 65: 6071-6079.

Network TCGA. 2008. Comprehensive genomic characterization defines human glioblastoma genes and core pathways. Nature 455: 10611068.

Network TR. 2011. Integrated genomic analyses of ovarian carcinoma. Nature 474: 609-615.

Nowell PC. 1976. The clonal evolution of tumor cell populations. Science 194: $23-28$.

Olcaydu D, Harutyunyan A, Jager R, Berg T, Gisslinger B, Pabinger I, Gisslinger H, Kralovics R. 2009. A common JAK2 haplotype confers susceptibility to myeloproliferative neoplasms. Nat Genet 41: 450-454.

Paques F, Haber JE. 1999. Multiple pathways of recombination induced by double-strand breaks in Saccharomyces cerevisiae. Microbiol Mol Biol Rev 63: 349-404.

Paris PL, Andaya A, Fridlyand J, Jain AN, Weinberg V, Kowbel D, Brebner JH, Simko J, Watson JE, Volik S, et al. 2004. Whole genome scanning identifies genotypes associated with recurrence and metastasis in prostate tumors. Hum Mol Genet 13: 1303-1313.

Rastas P, Koivisto M, Mannila H, Ukkonen E. 2005. A hidden Markov technique for haplotype reconstruction. Lect Notes Comput Sci 3692: 140-151.

Robinson MD, Smyth GK. 2008. Small-sample estimation of negative binomial dispersion, with applications to SAGE data. Biostatistics 9: 321332.

Robinson MD, McCarthy DJ, Smyth GK. 2010. edgeR: A Bioconductor package for differential expression analysis of digital gene expression data. Bioinformatics 26: 139-140.

Ronaghi M, Karamohamed S, Pettersson B, Uhlen M, Nyren P. 1996. Realtime DNA sequencing using detection of pyrophosphate release. Anal Biochem 242: 84-89.

Scheet P, Stephens M. 2006. A fast and flexible statistical model for largescale population genotype data: Applications to inferring missing genotypes and haplotypic phase. Am J Hum Genet 78: 629-644.

Solinas-Toldo S, Lampel S, Stilgenbauer S, Nickolenko J, Benner A, Dohner H, Cremer T, Lichter P. 1997. Matrix-based comparative genomic hybridization: Biochips to screen for genomic imbalances. Genes Chromosomes Cancer 20: 399-407.

Song H, Ramus SJ, Kjaer SK, DiCioccio RA, Chenevix-Trench G, Pearce CL, Hogdall E, Whittemore AS, McGuire V, Hogdall C, et al. 2009. Association between invasive ovarian cancer susceptibility and 11 best candidate SNPs from breast cancer genome-wide association study. Hum Mol Genet 18: 2297-2304.

Stadler ZK, Gallagher DJ, Thom P, Offit K. 2010a. Genome-wide association studies of cancer: Principles and potential utility. Oncology (Williston Park) 24: 629-637 .

Stadler ZK, Thom P, Robson ME, Weitzel JN, Kauff ND, Hurley KE, Devlin V, Gold B, Klein RJ, Offit K. 2010b. Genome-wide association studies of cancer. J Clin Oncol 28: 4255-4267.

Stephens M, Smith NJ, Donnelly P. 2001. A new statistical method for haplotype reconstruction from population data. Am J Hum Genet 68: 978-989.

Sun J, Liu W, Adams TS, Li X, Turner AR, Chang B, Kim JW, Zheng SL, Isaacs WB, Xu J. 2007a. DNA copy number alterations in prostate cancers: A combined analysis of published CGH studies. Prostate 67: 692-700.

Sun S, Greenwood CM, Neal RM. 2007b. Haplotype inference using a Bayesian hidden Markov model. Genet Epidemiol 31: 937-948.

Thorgeirsson TE, Geller F, Sulem P, Rafnar T, Wiste A, Magnusson KP, Manolescu A, Thorleifsson G, Stefansson H, Ingason A, et al. 2008. A variant associated with nicotine dependence, lung cancer, and peripheral arterial disease. Nature 452: 638-642.

Tuch BB, Laborde RR, Xu X, Gu J, Chung CB, Monighetti CK, Stanley SJ, Olsen KD, Kasperbauer JL, Moore EJ, et al. 2010. Tumor transcriptome sequencing reveals allelic expression imbalances associated with copy number alterations. PLOS ONE 5: e9317. doi: 10.1371/journal.pone. 0009317.

Turnbull C, Ahmed S, Morrison J, Pernet D, Renwick A, Maranian M, Seal S, Ghoussaini M, Hines S, Healey CS, et al. 2010. Genome-wide association 
study identifies five new breast cancer susceptibility loci. Nat Genet 42: 504-507.

Tuzun E, Sharp AJ, Bailey JA, Kaul R, Morrison VA, Pertz LM, Haugen E, Hayden H, Albertson D, Pinkel D, et al. 2005. Fine-scale structural variation of the human genome. Nat Genet 37: 727-732.

Wang DG, Fan JB, Siao CJ, Berno A, Young P, Sapolsky R, Ghandour G, Perkins N, Winchester E, Spencer J, et al. 1998. Large-scale identification, mapping, and genotyping of single-nucleotide polymorphisms in the human genome. Science 280: 1077-1082.

Witte JS. 2010. Genome-wide association studies and beyond. Annu Rev Public Health 31: 9-20.

Wooster R, Bignell G, Lancaster J, Swift S, Seal S, Mangion J, Collins N, Gregory S, Gumbs C, Micklem G. 1995. Identification of the breast cancer susceptibility gene BRCA2. Nature 378: 789-792.

Wu X, Hildebrandt MA, Chang DW. 2009. Genome-wide association studies of bladder cancer risk: A field synopsis of progress and potential applications. Cancer Metastasis Rev 28: 269-280.

Xie C, Tammi MT. 2009. CNV-seq, a new method to detect copy number variation using high-throughput sequencing. BMC Bioinformatics 10: 80. doi: 10.1186/1471-2105-10-80.
Yoon S, Xuan Z, Makarov V, Ye K, Sebat J. 2009. Sensitive and accurate detection of copy number variants using read depth of coverage. Genome Res 19: 1586-1592.

Zanke BW, Greenwood CM, Rangrej J, Kustra R, Tenesa A, Farrington SM, Prendergast J, Olschwang S, Chiang T, Crowdy E, et al. 2007. Genomewide association scan identifies a colorectal cancer susceptibility locus on chromosome 8q24. Nat Genet 39: 989-994.

Zhang K, Li JB, Gao Y, Egli D, Xie B, Deng J, Li Z, Lee JH, Aach J, Leproust EM, et al. 2009. Digital RNA allelotyping reveals tissuespecific and allele-specific gene expression in human. Nat Methods 6: $613-618$.

Zhao X, Weir BA, LaFramboise T, Lin M, Beroukhim R, Garraway L, Beheshti J, Lee JC, Naoki K, Richards WG, et al. 2005. Homozygous deletions and chromosome amplifications in human lung carcinomas revealed by single nucleotide polymorphism array analysis. Cancer Res 65: 55615570 .

Received February 22, 2011; accepted in revised form November 1, 2011. 


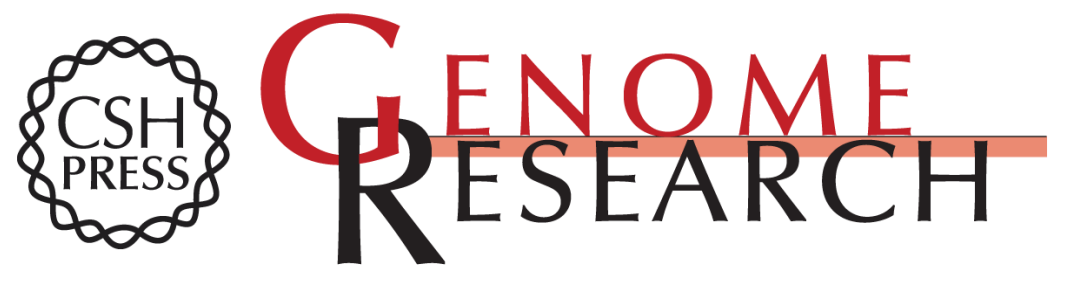

\section{Calling amplified haplotypes in next generation tumor sequence data}

Ninad Dewal, Yang Hu, Matthew L. Freedman, et al.

Genome Res. 2012 22: 362-374 originally published online November 16, 2011

Access the most recent version at doi:10.1101/gr.122564.111

Supplemental Material

References License

Email Alerting Service
http://genome.cshlp.org/content/suppl/2011/12/09/gr.122564.111.DC1

This article cites 91 articles, 22 of which can be accessed free at: http://genome.cshlp.org/content/22/2/362.full.html\#ref-list-1

Receive free email alerts when new articles cite this article - sign up in the box at the top right corner of the article or click here.

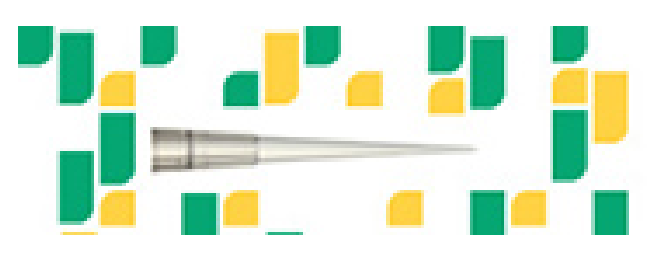

Focused on your science.

Jコగ

SCIENTIFIC

suos or seisnes

To subscribe to Genome Research go to: https://genome.cshlp.org/subscriptions 